\title{
Few-body bound state stability of dipolar molecules in two dimensions
}

\author{
A. G. Volosniev, D. V. Fedorov, A. S. Jensen, and N. T. Zinner \\ Department of Physics and Astronomy, Aarhus University, DK-8000 Aarhus C, Denmark
}

(Dated: November 20, 2018)

\begin{abstract}
Bound structures among dipolar molecules in multilayers are a topic of great interest in the light of recent experiments that have demonstrated the feasibility of the setup. While it is known that two molecules in two adjacent layers will always bind, larger complexes have only been scarcely addressed thus far. Here we prove rigorously that three- and four-body states will never be bound when the dipoles are oriented perpendicular to the layers. The technique employed is general and can be used for more molecules/layers and other geometries. Our analytical findings are supported by numerical calculations for both fermionic and bosonic molecules. Furthermore, we calculate the reduction in intralayer repulsion necessary to bind large complexes and estimate the influence of bound complexes in systems with many layers.
\end{abstract}

PACS numbers: 03.65.Ge, 36.20.-r, 67.85.-d

\section{INTRODUCTION.}

The production and manipulation of ultracold molecular gases is a truly riveting physical adventure at the moment [1. Interacting dipolar molecules confined to two dimensions are currently attracting substantial attention by way of its recent experimental realization and study of geometrical effects on chemical reactions [2]. Theoretically, this reduced dimensional setup has spurred immense interest and many promising proposals for novel many-body states exists [3. Many of these studies ignore or make rough guesses as to which few-body states are allowed in a setup with adjacent two-dimensional (2D) planes. An understanding of the basic building blocks is obviously crucial when trying to understand the complicated correlations in the many-body systems. The low-energy few-body spectrum with short-range interactions is well-known [4] and gives rise to the famous Efimov spectrum in three dimensions (3D) [5] and universal trimer states in 2D 6, 7]. However, the dipolar interactions is anisotropic and long-ranged and it is not clear that similar structures emerge.

A prominent example of the influence of bound states on the many-body physics is the BCS-BEC crossover in a two-component Fermi gas with attractive short-range interactions 8]. In the $2 \mathrm{D}$ case, the presence of a two-body bound dimer state plays a crucial role in the crossover 9 . This remains true for dipolar molecules in a bilayer with perpendicular polarization [10, and an effective dimerization of the system along these lines have been suggested in the multilayer setup [11. In the bilayer case, this can be infered from the dipolar potential which always supports a bound two-body state [12 15. However, there is a gap in our current knowledge of the stability of few-body states containing more than two molecules. In the present letter we give a complete characterization of the bound state structures for up to five layers with perpendicularly polarized dipoles (see Fig. 1).

The most stable three-body trimer system is a chain with one molecule in each layer where the pairwise interactions are attractive at short distances [16]. Two

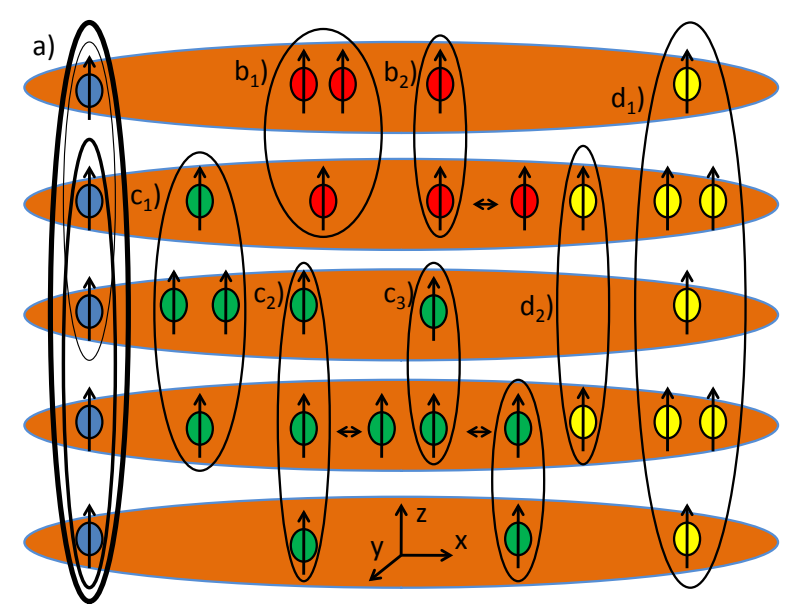

FIG. 1: (color online) Dipolar molecules in a multi-layer structure with dipoles oriented perpendicular to the planes. $a$ illustrates the different chains that are the most stable configurations. Three-body states are shown in $b_{1}$ and $b_{2}$, and four-body states in $c_{1}, c_{2}$, and $c_{3}$. $d_{1}$ shows a non-chain five layer configuration, while $d_{2}$ is a picture of a weakly bound dimer with twice the layer separation.

molecules in the same layer will repel each other. The most interesting trimer is therefore three molecules in two layers where two pairs can form bound subsystems whereas one pair is entirely repulsive $\left(b_{1}\right.$ in Fig. 11). The question is whether this trimer is bound with an energy below the dimer and a free molecule $\left(b_{2}\right.$ in Fig. 1). This is a special case in $2 \mathrm{D}$ of a repelling pair where each is attracted by a third particle. The four-body tetramer system with a fourth molecule in the layer next to the repelling pair ( $c_{1}$ in Fig. 1 is much closer to stability with five bound two-body subsystems and only one repelling pair. The question here is if that structure has a lower energy than a bound string of three molecules and a free molecule ( $c_{2}$ in Fig. 1) where only three bound subsystems remain. This of course implies that adding more molecules in favorable configurations could produce 
stable structures.

In the present letter we first rigourously prove that trimer and tetramer states are unstable for identical bosons or fermions in the layers. The mathematical method is very general and reduces the problem to a matter of geometrically identifying the threshold in the potential landscape of the systems. It can be applied to other systems where repulsive and attractive terms compete, like the study of excitons in carbon nanotubes [17, 18 or in organic semiconductors 19, 20. A suitable reduction of the in-plane dipolar repulsion leads to bound complexes as all interlayer interactions support bound states. We expect that this can be achieved in different ways, either by applying an electric field gradient perpendicular to the planes, by having different molecules in the system, or by direct manipulation of the dipole interaction in each layer through microwave transitions [21, 22]. Here we determine the critical repulsive strengths where stable structures emerge analytically and support our findings by detailed numerical calculations using the stochastic variational method [23].

\section{FORMULATION.}

The Schrödinger equation for $N$ particles with masses $m$ is

$$
\left(-\sum_{i=1}^{N} \frac{\hbar^{2}}{2 m} \vec{\nabla}_{i}^{2}+\sum_{i<j}^{N} \lambda_{i j} V_{i j}\left(\left|\vec{r}_{i}-\vec{r}_{j}\right|\right)\right) \Psi=E_{N} \Psi,
$$

with coordinates $\vec{r}_{i}$ and energy $E_{N}$. The interaction between particles $i$ and $j$ is $\lambda_{i j} V_{i j}$, i.e.

$$
V_{i j}(r, n)=\frac{\hbar^{2} d}{m} \frac{r^{2}-2 n^{2} d^{2}}{\left(r^{2}+n^{2} d^{2}\right)^{5 / 2}}, \lambda_{i j}=m \frac{D_{i} D_{j}}{\hbar^{2} d},
$$

where $d$ is the interlayer distance and $D_{i}$ is the dipole moment of molecule $i$. For $n=0$ we get the intralayer two-body $1 / r^{3}$ repulsion, while $n>0$ produces the interlayer potentials when the separation is $n d$. The strengths are defined by the product $D_{i} D_{j}$, and given through the dimensionless quantities $\lambda_{i j}$. The $V_{i j}(r, n=0)$ is modified at small distance by the transverse motion. This leads to the expression [24]

$$
V_{i j}(r, n=0)=\frac{\hbar^{2} d}{m} \frac{1}{2 \sqrt{2} l^{3}} U\left(\frac{3}{2}, 1, \frac{r^{2}}{2 l^{2}}\right),
$$

where $U$ is the confluent hypergeometric Kummer function and $l$ is the width of the layer. We have checked that our results are insensitive to $l$ in the interval $0.1 \leq l / d \leq$ 0.2 and the detailed properties at small distance are not important. Note that we assume that there is no tunneling between the layers, which implies that symmetrization is only relevant for particles in the same plane.

\section{A. Proof of instability.}

Consider first the three-body system with molecules 1 and 2 in the same plane and molecule 3 in an adjacent plane $\left(b_{1}\right.$ in Fig. 1). We assume that $D_{1}=D_{2}$ which may be different from $D_{3}$. We denote the attractive and repulsive strengths by $\lambda_{a} \equiv \lambda_{13}=\lambda_{23}$ and $\lambda_{r} \equiv \lambda_{12}$. Notice that both $\lambda_{a}$ and $\lambda_{r}$ are positive. The reason we talk of an attractive interaction in this case is that the potential always supports a bound state [14. We shall now prove that the system is unbound for $\lambda_{r}=\lambda_{a}$. Clearly, the system is bound for a sufficiently small ratio $\lambda_{r} / \lambda_{a}$. We can then, for any given $\lambda_{a}$, define a critical repulsive strength, $\lambda_{r}^{c r}\left[\lambda_{a}\right]$ (which is thus a function of $\lambda_{a}$ as indicated by the brackets), as the value where the energy precisely equals the two-body energy, $E_{2}\left[\lambda_{a}\right]$, of the bound 13 and 23 (interlayer) subsystems, i.e.

$$
\lambda_{r}^{c r}\left[\lambda_{a}\right]=\frac{E_{2}\left[\lambda_{a}\right]-\left\langle\Psi\left|T+\lambda_{a} V_{13}+\lambda_{a} V_{23}\right| \Psi\right\rangle}{\left\langle\Psi\left|V_{12}\right| \Psi\right\rangle},
$$

where $T$ is the kinetic energy operator and $\Psi$ is the normalized wave function at the threshold for binding. We now consider $F_{c r}=\frac{\partial}{\partial \lambda_{a}}\left(\frac{\lambda_{r}^{c r}\left[\lambda_{a}\right]}{\lambda_{a}}\right)$. We now make repeated use of the general fact that, for a Hamiltonian $h$ with eigenstate $|\phi\rangle$ and eigenvalue $\epsilon$, i.e. $h|\phi\rangle=\epsilon|\phi\rangle$, the partial derivative with respect to paramters that enter $h$ (and therefore also enter $\epsilon$ ) is $\epsilon^{\prime}=\left\langle\phi\left|h^{\prime}\right| \phi\right\rangle$. Applying this fact for the parameter $\lambda_{a}$, we obtain

$$
\frac{\partial \lambda_{r}^{c r}\left[\lambda_{a}\right]}{\partial \lambda_{a}}=\frac{\frac{\partial E_{2}\left[\lambda_{a}\right]}{\partial \lambda_{a}}-\left\langle\Psi\left|V_{13}+V_{23}\right| \Psi\right\rangle}{\left\langle\Psi\left|V_{12}\right| \Psi\right\rangle} .
$$

Defining the two-body bound state wave function, $\Phi$, and the related kinetic energy operator, $T_{2}$, we get

$$
\begin{aligned}
\lambda_{a}^{2} F_{c r} & =\frac{\lambda_{a} \frac{\partial E_{2}\left[\lambda_{a}\right]}{\partial \lambda_{a}}-E_{2}\left[\lambda_{a}\right]+\langle\Psi|T| \Psi\rangle}{\left\langle\Psi\left|V_{12}\right| \Psi\right\rangle} \\
& =\frac{\langle\Psi|T| \Psi\rangle-\left\langle\Phi\left|T_{2}\right| \Phi\right\rangle}{\left\langle\Psi\left|V_{12}\right| \Psi\right\rangle} .
\end{aligned}
$$

The structure of $\Psi$ is the product, $\Psi=\psi \Phi$, where $\psi$ only depends on the relative coordinate, $\mathbf{y}$, between the dimer, $\Phi$, and the third molecule. This follows from the $r^{-3}$ tail of the dipolar potential which is of shorter range than $r^{-2}$, which prohibits long-range correlations [4. The universal limit with a two-body cluster structure is therefore approached [25]. We immediately conclude that $\langle\Psi|T| \Psi\rangle-\left\langle\Phi\left|T_{2}\right| \Phi\right\rangle=\left\langle\psi\left|T_{y}\right| \psi\right\rangle$ is positive, where $T_{y}=T-T_{2}$ is the kinetic energy operator depending only on $\mathbf{y}$. Thus, $F_{c} r>0$ and the relative critical strength increases with $\lambda_{a}$.

We now compute $\lambda_{r}^{c r}\left[\lambda_{a}\right] / \lambda_{a}$ for $\lambda_{a}=\infty$, which is equivalent to infinite masses and negligibly small kinetic energies. The critical repulsive strength is found when the total potential in all space becomes larger than the two-body energy. Thus, the potential surface, 
$V_{13}+V_{23}+V_{12} \lambda_{r}^{c r}\left[\lambda_{a}\right] / \lambda_{a}$, depending on two relative coordinates, should have a minimum less than the minimum of the two-body potential in Eq. (2). This geometrical condition yields $\lambda_{r}^{c r}\left[\lambda_{a}\right] / \lambda_{a} \leq 0.373$. Since $F_{c r}>0$, this is a strict inequality for all finite values of $\lambda_{a}$. In particular, the system is unbound for all $\lambda_{a}$ when $\lambda_{r}=\lambda_{a}$.

The most stable four-body system is a chain with one molecule per layer (subset of $a$ in Fig. 1), followed by the $c_{1}$ configuration in Fig. 1. Four molecules in two layers are much less stable due to the additional repulsion. To study the stability when changing the repulsion, we consider two molecules in a single layer with $D_{2}=D_{3}$ and one molecules in each of the layers above and below it with $D_{1}=D_{4}$, keeping the product $D_{1} D_{3}$ fixed. The strengths are $\lambda_{a}=\lambda_{13}=\lambda_{12}=\lambda_{24}=\lambda_{34}, \lambda_{r}=\lambda_{23}$, and $\lambda_{14}=\lambda_{a}^{2} / \lambda_{r}$. The threshold structure, $\Psi$, approached when $\lambda_{r} \rightarrow \lambda_{r}^{c r}\left[\lambda_{a}\right]$, is three molecules in three layers, $\Phi$, and a free fourth molecule ( $c_{2}$ in Fig. 11). The condition of equal energies analogous to Eq. (4) becomes

$$
\begin{gathered}
\langle\Psi| T+\lambda_{r}^{c r}\left[\lambda_{a}\right] V_{23}+\frac{\lambda_{a}^{2}}{\lambda_{r}^{c r}\left[\lambda_{a}\right]} V_{14}+\lambda_{a}\left(V_{13}+V_{12}+V_{24}+V_{34}\right) \\
=\left\langle\Phi\left|T_{3}+\lambda_{a} V_{13}+\lambda_{a} V_{24}+\frac{\lambda_{a}^{2}}{\lambda_{r}^{c r}\left[\lambda_{a}\right]} V_{14}\right| \Phi\right\rangle .
\end{gathered}
$$

Similar to the three-body case, the threshold structure is $\Psi=\psi \Phi$, where $\psi$ is the wave function depending on the relative coordinate, $\mathbf{y}$, between the trimer and the fourth molecule. This means that $\left\langle\Psi\left|V_{14}\right| \Psi\right\rangle=\left\langle\Phi\left|V_{14}\right| \Phi\right\rangle$, implying that the critical value easily is found from Eq. (7). A completely analogous procedure as above yields

$$
\lambda_{a}^{2} F_{c r}=\frac{\langle\Psi|T| \Psi\rangle-\left\langle\Phi\left|T_{3}\right| \Phi\right\rangle}{\left\langle\Psi\left|V_{23}\right| \Psi\right\rangle},
$$

where $T_{3}$ is the three-body kinetic energy operator. This derivative is postive and an upper bound for stability is obtained from $\lambda_{a}=\infty$. The geometries of the potentials are such that $\lambda_{r}^{c r}\left[\lambda_{a}\right] / \lambda_{a} \leq 0.75$, and all these four-body systems in two or three layers are unstable.

Our results strongly indicate that additional layers could lead to stable configurations with more than one molecule in each layer. The proofs above hold for bosons and distinguishable particles. However, the threshold for fermions is always higher and produces less stable systems.

\section{NUMERICAL RESULTS.}

We illustrate our findings numerically using the stochastic variational method with correlated gaussians 23]. Fig. 2 shows two- and three-body energies as function of $\lambda_{r} / \lambda_{a}$ with fixed $\lambda_{a}=10$ for the $b_{1}$ configuration in Fig. 11. The trimer energies increase with increasing repulsion, and become unstable at the dimer threshold. Resonances could exist at higher energies but the effective barriers are tiny for these potentials. A large width would immediately be accumulated and the resonance disappears by merging into the two-body continuum background. The fermion energies exceed the boson

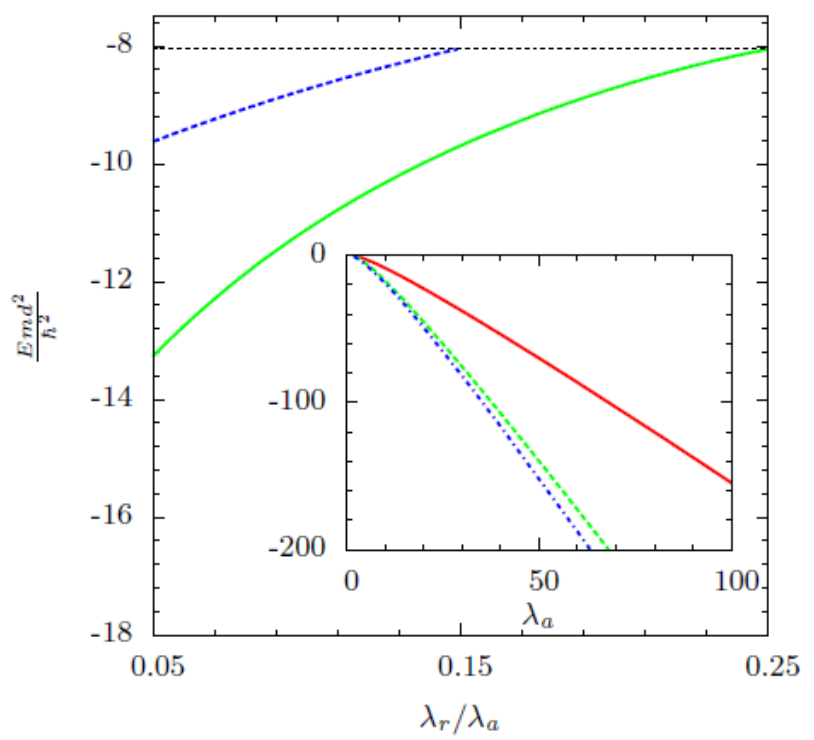

FIG. 2: (color online) Numerical trimer enegies as function of $\lambda_{r}^{\Psi} / \lambda_{a}=D_{1} / D_{3}$ of the configuration $b_{1}$ in Fig. 1 calculated with $\lambda_{a}=10$ for bosons (solid green) and fermions (dashed blue). A horizontal dashed marks the two-body threshold. The inset compares the two-layer dimer (solid red) with the three-layer trimer as function of $\lambda_{a}$ (dot-dashed blue). The (green) dashed curve is twice the dimer energy demonstrating that the three-layer trimer is more stable than two free dimers.

energies due to the antisymmetry which itself costs energy compared to symmetric systems. This is unavoidable in spite of the repulsion because the attraction at short distance must be larger to provide a bound state, implying that the two molecules in the same layer cannot be far apart. The distance from stability is reflected in the critical repulsion below which the system is bound. The lower curves in Fig. 3 show the numerical results for trimers. At large $\lambda_{a}$ the analytical results 0.373 is approached very slowly, while at small $\lambda_{a}$ only a small repulsive perturbation is needed to break the exponentially weakly bound system.

Consider now the four-body system in configuration $c_{1}$ of Fig. 1] As demonstrated in the inset of Fig. 2, a chain of three molecules in three layers is more bound that two dimers. This can be understood since it gains energy from the attractive interaction of configuration $d_{2}$ in Fig. 1. The threshold for binding $c_{1}$ is therefore a chain of three molecules and a free molecule, $c_{2}$, rather than $c_{3}$. The energies for the $c_{1}$ system are shown in Fig. 4 where we again see that the threshold is reached for $\lambda_{r} / \lambda_{a}<1$. The relative critical strengths are shown in Fig. 3, where the asymptotic strong interaction limit of 0.75 obtained from geometry is approached. 


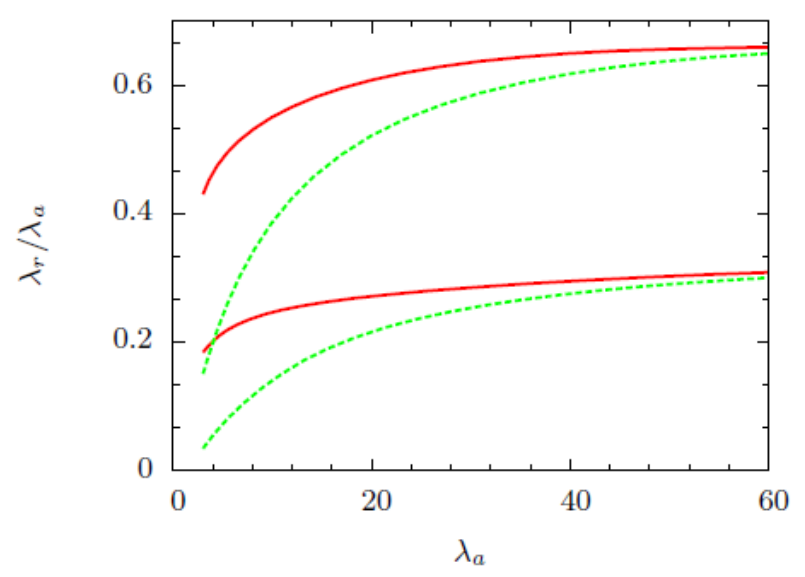

FIG. 3: (color online) The critical strengths, $\lambda_{r}^{c r} / \lambda_{a}$, as functions of $\lambda_{a}$, for configurations $b_{1}$ (lower curves) and $c_{1}$ (upper curves) of Fig. 1 with fermionic (solid) and bosonic (dashed) species. The asymptotic values, 0.373 and 0.75 , for large $\lambda_{a}$ are slowly approached.

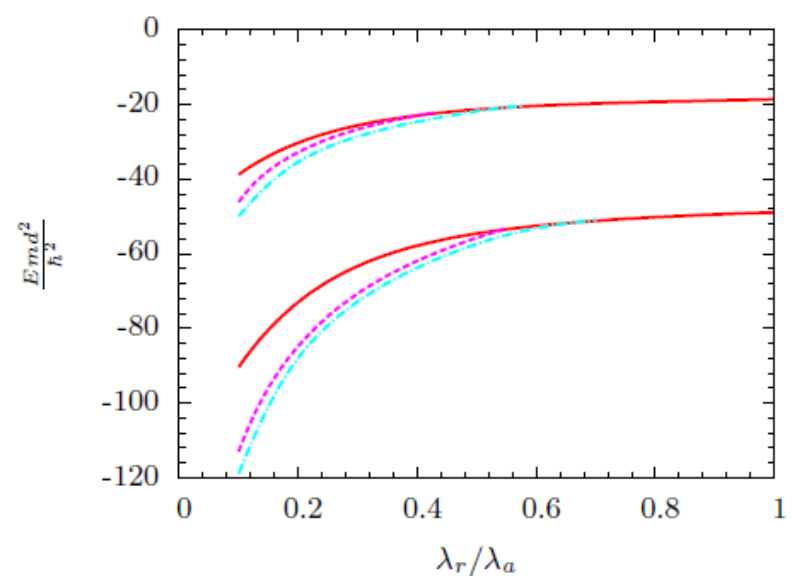

FIG. 4: (color online) Tetramer energies as function of $\lambda_{r} / \lambda_{a}$ in configuration $c_{1}$ of Fig. 1 with $\lambda_{a}=10$ (upper) and $\lambda_{a}=20$ for fermions (dashed purple) and bosons (dot-dashed blue). The solid red curve shows the threshold energy ( $c_{2}$ of Fig. 1 .

\section{A. Structure.}

The structure of trimer and tetramer is contained in relative distances within the bound state. In the strong dipole limit, all molecules remain close until the repulsion is extremely close to the threshold where one molecule eventually leaks out through the repulsive barrier. The universal limit of $\left\langle r^{2}\right\rangle|E| \rightarrow \frac{1}{3}$ is approached from below but only for very small energies [14, 25. For weak dipoles, the wave functions are already distributed over large regions of space. Our numerical calculations confirm these expectations that are used in the proof of in- stability.

IV. CONCLUSIONS AND PERSPECTIVES.

We have demonstrated that in two or three layer system with perpendicularly polarized dipolar molecules, no three- or four-body bounds states are stable. This was shown rigorously through a novel analytical approach that predicts geometrically how much the repulsive interactions in the system must be reduced to stabilize such states. We also presented numerical results in support of our conclusions and predicting exact critical values for when bound state with more than two molecules are stable in the setup. Fermionic systems are found to be less stable than bosonic for all systems studied. Here we have studied the case of perpendicular polarization, but we note that similar arguments can be applied for moderate tilting angles where the in-plane interaction remains repulsive on average.

While we find that chains are the most stable configurations ( $a$ in Fig. 1), our results show that four-body states are much closer to stability than three-body states. This implies that an increase in the number of layers can lead to bound complexes with more than one molecule per layer (as for instance shown in configuration $d_{1}$ of Fig. 1). Disregarding these few-body states, both density wave 26] and crystalline [27] phases have been predicted in the multilayer setup. With a richer spectrum of few-body complexes, we expect that more complicated, stable many-body configurations are possible when the complexes discussed here are used as building blocks. Detecting the structures experimentally should be possible by several techniques such as RF spectroscopy [28], lattice shaking [29, or in situ optical detection 30, 31.

As demonstrated here, one can tune bound state stability by reducing the intra-layer repulsion. If an electric field gradient is applied that is steep enough to make the interaction in adjacent layer different the effective repulsion could be reduced and stabilize fewbody bound states. Alternatively, one can imagine driving microwave transitions among rotational states in the molecules [21, 22] into states of different effective dipole moment for a given external dc field. Through this technique it might be possible to even reverse the direction of the effective dipole moment, yielding a potential that is repulsive at short distance and attractive in the tail. This will produce entirely new structures and is an exciting direction for future studies.

\section{Acknowledgments}

We thank J. Arlt, J. F. Sherson, and B. Wunsch for enlightening discussions.
[1] K. Winkler et al., Phys. Rev. Lett. 98, 043201 (2007); S. Ospelkaus et al., Nature Phys. 4, 622 (2008); J. G. Danzl et al., Science 321, 1062 (2008); K. K. Ni et al., Science 
322, 231 (2008); J. Deiglmayr et al., Phys. Rev. Lett. 101, 133004 (2008); F. Lang, K. Winkler, C. Strauss, R. Grimm, and J. Hecker Denschlag, Phys. Rev. Lett. 101, 133005 (2008); S. Ospelkaus et al., Science 327, 853 (2010); K. K. Ni et al., Nature 464, 1324 (2010). J. G. Danzl et al., Nature Phys. 6, 265 (2010).

[2] M. H. G. de Miranda et al., Nature Phys. 7, 502 (2011).

[3] M. A. Baranov, Phys. Rep. 464, 71 (2008); T. Lahaye, C. Menotti, L. Santos, M. Lewenstein, and T. Pfau, Rep. Prog. Phys. 72, 126401 (2009); L. D. Carr et al., New J. Phys. 11, 055049 (2009).

[4] A.S. Jensen, K. Riisager, D.V. Fedorov and E. Garrido, Rev. Mod. Phys. 76 (2004) 215-261.

[5] F. Ferlaino and R. Grimm, Physics 3, 9 (2010).

[6] E. Nielsen, D. V. Fedorov, A. S. Jensen, Phys. Rev. A 56, 3287 (1997).

[7] F. F. Bellotti et al., J. Phys. B: At. Mol. Opt. Phys. 44, 205302 (2011); F. F. Bellotti et al., Phys. Rev. A 85, 025601 (2012).

[8] I. Bloch, J. Dalibard, and W. Zwerger, Rev. Mod. Phys. 80, 885 (2008); S. Giorgini, L. P. Pitaevskii, and S. Stringari, Rev. Mod. Phys. 80, 1215 (2008).

[9] M. Randeria, J.-M. Duan, and L.-Y. Shieh, Phys. Rev. Lett. 62, 981 (1989); Phys. Rev. B 41, 327 (1990).

[10] A. Pikovski, M. Klawunn, G. V. Shlyapnikov, and L. Santos, Phys. Rev. Lett. 105, 215302 (2010); N. T. Zinner, B. Wunsch, D. Pekker, and D.-W. Wang, Phys. Rev. A 85, 013603 (2012).

[11] A. C. Potter, E. Berg, D.-W. Wang, B. I. Halperin, and E. Demler, Phys. Rev. Lett. 105, 220406 (2010).

[12] S.-M. Shih and D.-W. Wang, Phys. Rev. A 79, 065603 (2009).

[13] M. Klawunn, A. Pikovski, and L. Santos, Phys. Rev. A 82, 044701 (2010).

[14] A. G. Volosniev, D. V. Fedorov, A. S. Jensen, and N. T. Zinner, Phys. Rev. Lett. 106 (2011), 250401.

[15] N. T. Zinner, J. R. Armstrong, A. G. Volosniev, D. V. Fedorov, and A. S. Jensen, arXiv:1105.6264v1.
[16] J. R. Armstrong, N. T. Zinner, D. V. Fedorov, and A. S. Jensen, arXiv:1106.2102 2 .

[17] J. Deslippe, C. D. Spataru, D. Prendergast, and S. G. Louie, Nano Lett. 7, 1626 (2007).

[18] R. Matsunaga, K. Matsuda, and Y. Kanemitsu, Phys. Rev. Lett. 106, 037404 (2011).

[19] D. G. Lidzey et al., Nature 395, 53 (1998).

[20] X.-Y. Zhu, Q. Yang, and M. Muntwiler, Acc. Chem. Res. 42, 1779 (2009).

[21] A. Micheli, G. Pupillo, H. P. Büchler, and P. Zoller, Phys. Rev. A 76, 043604 (2007).

[22] D. DeMille, Phys. Rev. Lett. 88, 067901 (2002).

[23] A. G. Volosniev et al., J. Phys. B 44, 125301 (2011).

[24] J. C. Cremon, G. M. Bruun, and S. M. Reimann, Phys. Rev. Lett. 105, 255301 (2010).

[25] J. R. Armstrong, N. T. Zinner, D. V. Fedorov, A. S. Jensen, Europhys. Lett. 91, 16001 (2010).

[26] K. Sun, C. Wu, and S. Das Sarma, Phys. Rev. B 82, 075105 (2010); Y. Yamaguchi, T. Sogo, T. Ito, and T. Miyakawa, Phys. Rev. A 82, 013643 (2010); N. T. Zinner and G. M. Bruun, Eur. Phys. J. D 65, 133 (2011); M. Babadi and E. Demler, Phys. Rev. B 84, 235124 (2011); L. M. Sieberer and M. A. Baranov, Phys. Rev. A 84, 063633 (2011); M. M. Parish and F. M. Marchetti, arXiv:1109.2464 1; J. K. Block, N. T. Zinner, and G. M. Bruun, in preparation.

[27] C. Mora, O. Parcollet, and X. Waintal, Phys. Rev. B 76, 064511 (2007); H. P. Büchler et al., Phys. Rev. Lett. 98, 060404 (2007); G. E. Astrakharchik, J. Boronat, I. L. Kurbakov, and Yu. E. Lozovik, Phys. Rev. Lett. 98, 060405 (2007).

[28] Y. Shin, C. H. Schunck, A. Schirotzek, and W. Ketterle, Phys. Rev. Lett. 99, 090403 (2007).

[29] N. Strohmaier et al., Phys. Rev. Lett. 104, 080401 (2010).

[30] B. Wunsch et al., Phys. Rev. Lett. 107, 073201 (2011).

[31] N. T. Zinner et al., Phys. Rev. A 84, 063606 (2011). 